\title{
PD-L1 Expression Predicts a Distinct Prognosis in Krukenberg Tumor with Corresponding Origins
}

\author{
Haiyan Tai $\mathbb{D}^{1},{ }^{1}$ Qin Yang, ${ }^{2}$ Zhiyong Wu, ${ }^{1}$ Su'an Sun, ${ }^{3}$ Rui Cao, ${ }^{4}$ Yanfeng Xi, ${ }^{5}$ Ran Zhao, \\ Mengyu Zhang, ${ }^{6}$ Zhigang Zhang $\left(\mathbb{1},{ }^{2}\right.$ and Congjian $X u \mathbb{B}^{1,7,8}$ \\ ${ }^{1}$ Obstetrics and Gynecology Hospital, Fudan University, Shanghai, China \\ ${ }^{2}$ State Key Laboratory of Oncogenes and Related Genes, Shanghai Cancer Institute, Shanghai Jiao Tong University, Shanghai, China \\ ${ }^{3}$ Department of Pathology, The First Hospital of Huai'an City, Nanjing Medical University, Huai'an, Jiangsu Province, China \\ ${ }^{4}$ Dalian Obstetrics and Gynecology Hospital, Dalian, Liaoning Province, China \\ ${ }^{5}$ Department of Pathology, Shanxi Provincial Cancer Hospital, Shanxi Medical University, Taiyuan, Shanxi, China \\ ${ }^{6}$ Department of Gynecology, 411 Military Hospital, Shanghai, China \\ ${ }^{7}$ Department of Obstetrics and Gynecology of Shanghai Medical School, Fudan University, Shanghai, China \\ ${ }^{8}$ Shanghai Key Laboratory of Female Reproductive Endocrine Related Diseases, Shanghai, China
}

Correspondence should be addressed to Zhigang Zhang; zzhang@shsci.org and Congjian Xu; xucongjian@fudan.edu.cn

Received 21 February 2018; Accepted 4 April 2018; Published 8 May 2018

Academic Editor: Jian Song

Copyright (c) 2018 Haiyan Tai et al. This is an open access article distributed under the Creative Commons Attribution License, which permits unrestricted use, distribution, and reproduction in any medium, provided the original work is properly cited.

\begin{abstract}
Krukenberg tumor (KT) is an uncommon ovarian metastatic signet-ring cell adenocarcinoma that mostly metastasizes from gastrointestinal carcinoma. Optimal treatment options for KTs are limited. Programmed death-1 (PD-1)/programmed death-ligand 1 (PD-L1) inhibitors have shown remarkable activity in clinical trials for metastatic tumors. Here, we evaluated PD-L1 expression and T cell infiltration in KTs and their corresponding primary tumors. Positive tumor PD-L1 expression was detected in 9 (25.7\%) KTs from gastric carcinomas (GCs) and in 20 (66.7\%) KTs from colorectal carcinomas (CRCs). Patient survival was assessed according to the PD-L1 status and CD8 ${ }^{+} \mathrm{T}$ cell density. Positive tumor PD-L1 expression in KTs from GCs was associated with poor prognosis. In contrast, positive tumor PD-L1 expression in KTs from CRCs was associated with an improved prognosis. We analyzed copy number variations of the PD-L1 gene in KTs. PD-L1 expression was higher in cases with copy number gains. The T cell densities within KTs and their corresponding primary tumors were compared. The densities of $\mathrm{CD}^{+} \mathrm{T}$ cells correlated significantly between the primary tumors and KTs from the same case. Taken together, the research further highlighted targets for immune-based therapy in KTs from GCs and CRCs.
\end{abstract}

\section{Introduction}

Ovarian metastatic tumors that contain a component of signet-ring cells are known as Krukenberg tumors (KTs) and originate mainly from the stomach $(76 \%)$, intestines (11\%), breast (4\%), and other organs [1]. KTs chiefly affect premenopausal women. Although a few studies have suggested that patients might benefit from metastasectomy with systemic chemotherapy, optimal treatment options are limited and the prognosis is poor [2-4]. Most patients die within 2 years (median survival time: 14 months) [5]. Thus, the need for new strategies to treat KTs is pressing.

Immunotherapy has emerged as a promising measure for cancer treatment [6]. Accumulative data have revealed the successful application of immune checkpoint blockers in multiple cancer types, including advanced gastrointestinal carcinoma [7-10]. In one clinical trial, high response rates to PD-1 inhibitors were observed among advanced colorectal carcinoma (CRC) and gastric carcinoma (GC) patients whose tumors were mismatch repair-deficient [10]. The 
relationship between PD-L1/PD-1 expression and therapeutic response was evident, and none of the PD-L1-negative tumors responded [11]. Thus, a study of tumor PD-L1 expression in ovarian metastases from gastrointestinal cancer is needed.

We investigated PD-L1 expression in KTs from GCs and CRCs, examined the correlation between PD-L1 expression and $\mathrm{T}$ cell infiltration, and evaluated the impact of PD-L1 expression on prognosis. Copy number variations in the $P D-L 1$ gene in KTs from GCs were analyzed. The immune microenvironment of KTs was also assessed and compared with that of the primary tumor from the same case.

\section{Materials and Methods}

2.1. Case Cohort. We reviewed a retrospective cohort study of 65 cases. A tissue microarray was constructed for paraffin sample tissues and included $35 \mathrm{KT}$ s with 23 matched primary GCs and 30 KTs with 28 matched primary CRCs collected at Fudan University Affiliated Obstetrics and Gynecology Hospital and cooperative hospitals between 2000 and 2015. The overall survival (OS) time was defined as the interval between the ovarian metastasectomy operation and death or survival. Exclusion criteria included (a) the absence of surgery of KT and (b) the validation of an ovarian nonadenocarcinoma metastasis. Samples and medical records were approved by the research ethics committee of Fudan University Affiliated Obstetrics and Gynecology Hospital and cooperative hospitals.

2.2. Immunohistochemistry. The primary antibodies used were as follows: anti-PD-L1 for immunohistochemistry (IHC, rabbit monoclonal antibody, Abcam, UK, ab205921, $1: 100$ ), anti-PD-L1 for multiplex IHC (rabbit monoclonal antibody, CST, USA, 78701, 1:200), anti-PD-1 (mouse monoclonal antibody, CST, USA, 43248, 1:100), antiFOXP3 (rabbit monoclonal antibody, CST, USA, 98377, 1:50), anti-CD3 (rabbit monoclonal antibody, Abcam, UK, ab16669, 1:100), anti-CD8 (mouse monoclonal antibody, Abcam, UK, ab11147, 1:25), and anti-CD8 for multiplex IHC (mouse monoclonal antibody, CST, USA, 78701, 1:250). Positive staining was visualized with DAB substrate liquid (CST, USA), and counterstaining was performed with hematoxylin. Scoring was performed by two senior pathologists.

Tumor PD-L1 expression was determined by the presence of membrane staining in tumor cells as previously reported [12]. Stromal PD-L1 expression was evaluated according to the presence of membrane staining in stromal cells. The PD $1^{+}, \mathrm{FOXP}^{+}, \mathrm{CD}^{+}$, and $\mathrm{CD}^{+}$cell densities $\left(\right.$ cells $/ \mathrm{mm}^{2}$ ) were quantified using digital image analysis. The $\mathrm{CD}^{+} \mathrm{T}$ cell densities were dichotomized into "high" and "low" groups according to the median.

2.3. Multiplex Immunochemistry. The tissue sections were deparaffinized, rehydrated, and incubated with $0.3 \%$ hydrogen peroxide, and the antigen was unmasked in $10 \mathrm{mM}$ sodium citrate buffer using a microwave. After the sections were incubated with the primary antibody for $45 \mathrm{~min}$ at RT, slides were incubated with anti-rabbit secondary antibody (NEF812E001EA; PerkinElmer) or anti-mouse secondary antibody (NEF822E001EA; PerkinElmer) for $30 \mathrm{~min}$ at RT. Tyramide (TSA) plus fluorescein (NEL741E001KT, PerkinElmer) or TSA plus Cyanine 5 (NEL745E001KT, PerkinElmer) was added to the slides at a 1:50 dilution, and the slides were incubated for $10 \mathrm{~min}$ at RT. A microwave was used to remove the combined antibody. TSA plus fluorescein or TSA plus Cyanine 5 was added to slides at a 1:50 dilution. Cell nuclei were counterstained with DAPI. The slides were imaged using Vectra imaging software.

2.4. Copy Number Analysis. The PD-L1 copy number in 13 paraffin samples was analyzed using the OncoScan formalin-fixed and paraffin-embedded (FFPE) Assay Kit (Affymetrix; USA). Total FFPE DNA was extracted using the QIAamp DNA Mini Kit (Qiagen; Germany). Results were analyzed using ChAS (Chromosome Analysis Suite, version 3.1.1.27) software. Raw data was submitted to ArrayExpress, and the data accession number is E-MTAB-6277.

2.5. Statistical Analysis. Statistical analyses were conducted using Statistical Product and Service Solutions 16.0 software (Chicago, IL, USA). Chi-square tests in cross tables were performed to examine the correlation between the PD-L1 expression levels and clinical parameters or lymphocyte variables. Correlations of $\mathrm{T}$ cell infiltration between the primary tumor and metastases were calculated with the Spearman correlation test. OS was estimated using the Kaplan-Meier method, and differences were evaluated using the log-rank test. Graphic representations were analyzed with GraphPad Prism software (San Diego, CA). $P$ values $<0.05$ were considered to be statistically significant.

\section{Results}

3.1. Clinicopathological Findings. A retrospective cohort study of 65 patients, including 35 KTs from GCs and CRCs, was conducted. The clinicopathological characteristics of the patients are detailed (Supplementary Tables S1 and S2). The median age of the patients with KTs from GCs was 41 (22-62) years. The median OS time was 9 months $(9 \pm 1.7$ months). Among the 35 cases, tumors were more common in premenopausal women $(82.9 \%)$ than in postmenopausal women $(17.1 \%)$. Most patients $(82.9 \%)$ had synchronous ovarian metastasis. The median age of the patients with KTs from CRCs was 50 (30-79) years. The median OS time was 17 months $(17 \pm 2.397)$. Among the 30 cases, postmenopausal women $(53.3 \%)$ and premenopausal women (46.7\%) were equally represented.

3.2. Expression of PD-L1 in KTs and Its Association with Clinicopathological Parameters. PD-L1 was expressed on the cell membranes of the tumor cells or stromal cells (Figure 1(a)). Tumor PD-L1 expression was detected in 9 (25.7\%) KTs from GCs and $20(66.7 \%)$ KTs from CRCs. Stromal PD-L1 expression was detected in 4 (11.4\%) KTs from GCs and 8 (26.7\%) KTs from CRCs (Figure 1(c)). The expression of the tumor and stromal PD-L1s was evaluated by multiplex IHC techniques (Figure 1(b)). 

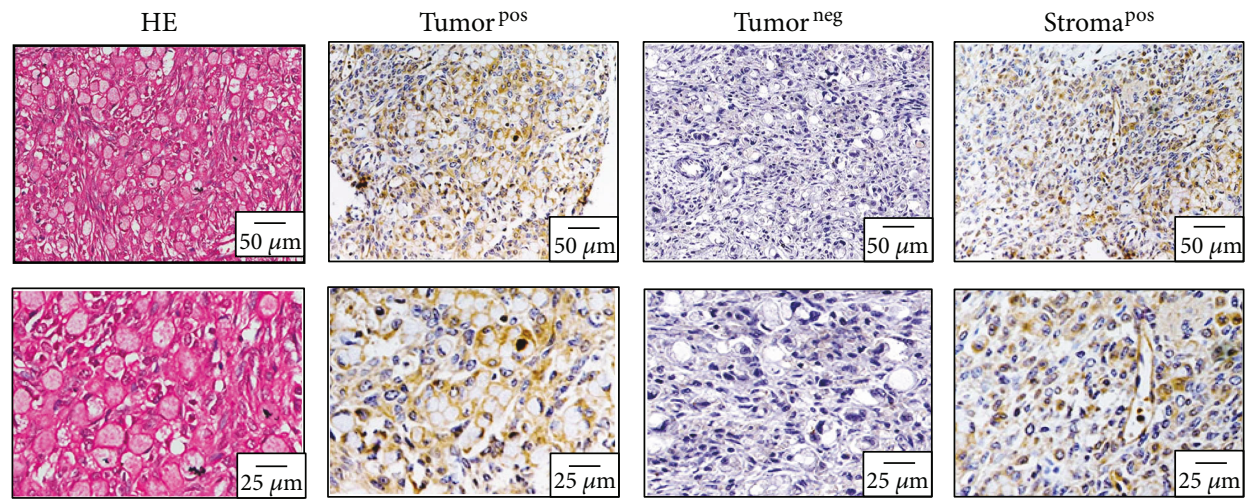

(a)

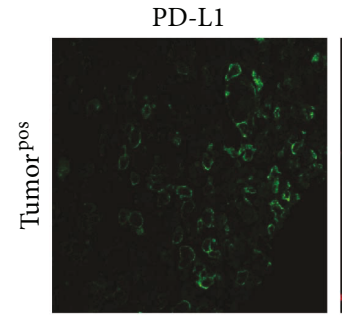

CD8

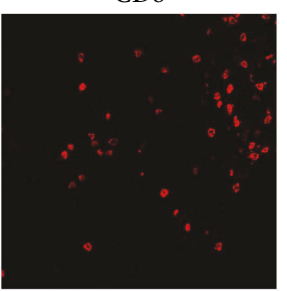

DAPI
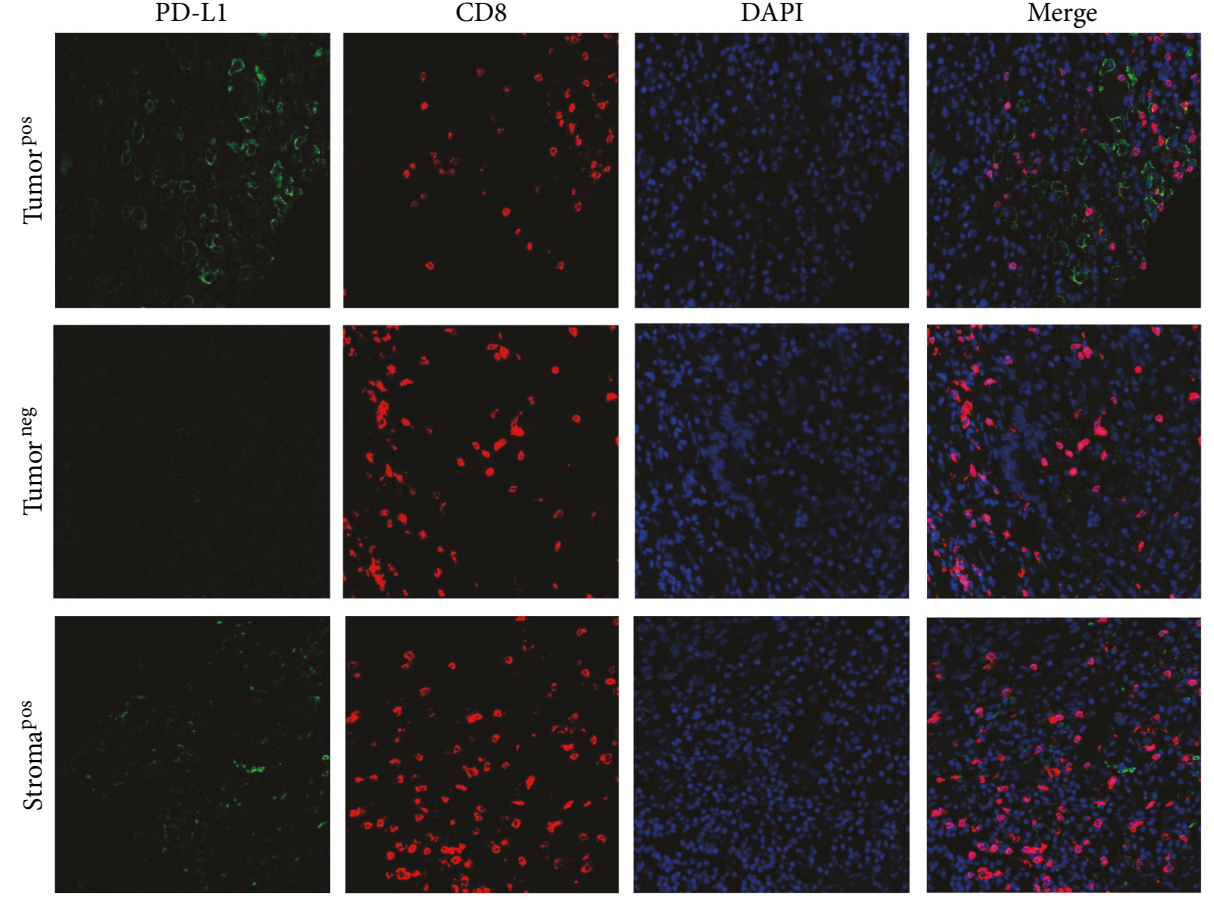

(b)

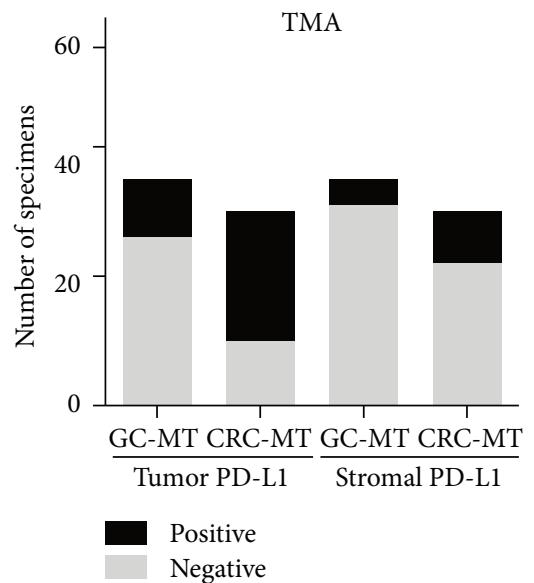

(c)

FIgURE 1: PD-L1 expression in KTs. (a) Representative samples of HE and IHC staining of PD-L1 were shown, including tumor cells, stromal cells, and PD-L1-negative tumors. (b) Multiplex IHC of PD-L1 and CD8 on tumor cells and stromal cells. Nuclei were stained with DAPI. (c) The percentage of the tissue displaying positive and negative tumor and immune stromal PD-L1 staining in KTs from the GCs and CRCs. MT: metastasis. 
The relationship between the tumor or stromal PD-L1 expression and the clinical characteristics of the patients with ovarian metastasis are shown (Supplementary Tables S1 and S2). In the GC patients, we observed that the tumor PD-L1 expression was positively associated with postmenopausal status $(P=0.027)$. In the CRC patients, an inverse relationship between the tumor PD-L1 expression and the extent of signet-ring cells was observed $(P=0.009)$. No significant relationship was evident between the stromal PD-L1 expression and any clinicopathological feature (Supplementary Tables S1 and S2).

3.3. PD-L1 Expression and $C D 8^{+} T$ Cell Infiltration Are Associated with Overall Survival. Kaplan-Meier analysis was performed to evaluate OS according to the tumor or stromal PD-L1 expression in KTs (Figures 2 and 3). Positive tumor PD-L1 expression in KTs from GCs was associated with worse OS than negative tumor PD-L1 expression $(P=0.008$, Figure $2(\mathrm{c}))$. Conversely, positive tumor PD-L1 expression in KTs from CRCs was associated with an improved OS compared to negative tumor PD-L1 expression $(P=0.025$, Figure $3(\mathrm{c}))$.

The impact of tumor PD-L1 expression in primary tumors on OS was also determined. Positive tumor PD-L1 expression had a tendency for poor prognosis in the GC cases $(P=0.142$, Figure 2(b) ) and for an improved prognosis in the CRC cases compared to negative tumor PD-L1 expression $(P=0.118$, Figure $3(\mathrm{~b}))$.

Because PD-L1/PD-1 signaling might be secondary to $\mathrm{CD}^{+} \mathrm{T}$ cell infiltration [13], we quantified $\mathrm{CD}^{+} \mathrm{T}$ cell infiltration in the ovarian metastases. However, no significant correlation was evident between the tumor or stromal PD-L1 expression and the $\mathrm{CD}^{+}$density (Supplementary Tables S3 and S4). Kaplan-Meier analysis was performed to evaluate OS according to the density of $\mathrm{CD}^{+} \mathrm{T}$ cells in the ovarian metastases. High densities of infiltrating $\mathrm{CD}^{+} \mathrm{T}$ cells were associated with prolonged $\mathrm{OS}$ in the GC ovarian metastases $(P=0.182$, Figure 2(e) $)$ and in the CRC ovarian metastases $(P=0.037$, Figure $3(\mathrm{e}))$. For the CRC patients, the combination of the positive tumor PD-L1 expression and a high $\mathrm{CD}^{+} \mathrm{T}$ cell density was associated with a better OS rate than either factor alone $(P=0.002$, Figure $3(\mathrm{~g}))$.

Characteristics including PD-L1 expression in ovarian metastases were analyzed using COX proportion hazard regression models to assess the prognostic values (Table 1). In GC patients, the positive tumor PD-L1 expression $(\mathrm{HR}=3.201$ (95\% CI: 1.273-8.050), $P=0.013)$ and menopause status ( $\mathrm{HR}=2.860$ (95\% CI: $1.057-7.736), P=0.038)$ were analyzed to be risk factors for OS.

In CRC patients, the negative tumor PD-L1 expression in metastases $(\mathrm{HR}=5.129$ (95\% CI: $1.567-16.791), P=0.007)$ and high ratio of signet cells ( $\mathrm{HR}=4.655$ (95\% CI: $1.366-$ $15.863), P=0.014)$ were identified as potential prognostic factors (Table 2). The negative tumor PD-L1 expression retained its significance in the multivariate analysis.

3.4. Copy Number Variation of PD-L1 and Its Association with PD-L1 Expression. Aiming to evaluate the impact of copy number alterations on PD-L1 expression, we performed a copy number analysis of 13 KTs from GCs with the OncoScan FFPE assay adjusted to 13 normal control tissues. The copy number in each case was analyzed, and the results revealed $P D$ - L1 gene copy number gains in 2 cases (15.4\%), $P D-L 1$ gene copy number losses in 2 cases (15.4\%), and a normal copy number status in 9 cases $(69.2 \%)$. The PD-L1 expression was higher in cases with copy number gains than in normal cases $(P=0.0209)$ or cases with copy number losses $(P=0.0101)$ (Figure 4$)$.

3.5. Association of PD-L1 Status with T Cell Infiltration. Although DNA copy number gains play an important role in gene overexpression, the evidence explaining the deregulation of PD-L1 was insufficient. The immunological microenvironment is reportedly involved in tumor PD-L1 upregulation [13]. The associations between tumor PDL1 expression and T cell densities in KTs were analyzed. The tumor PD-L1 expression score was not significantly associated with $\mathrm{CD}^{+}, \mathrm{CD}^{+}, \mathrm{FOXP}^{+}$, or $\mathrm{PD}-1^{+} \mathrm{T}$ cell infiltration (Supplementary Tables S3 and S4).

3.6. T Cell Infiltration in Primary Tumor and KTs. We compared T cell densities in the primary GCs to those in the KTs from the same individuals $(n=23)$. The densities of $\mathrm{CD}^{+}$, $\mathrm{CD}^{+}$, and $\mathrm{FOXP}^{+}$cells were significantly higher in the primary GCs than in the KTs $(P<0.001$; Figure $5(\mathrm{~b}))$. Furthermore, the densities of $\mathrm{CD}^{+}$cells were positively correlated between the primary tumors and KTs $(r=0.438$, $P=0.047$, Figure 5(d)). However, the densities of $\mathrm{CD}^{+}$and $\mathrm{FOXP}^{+}$cells were not correlated between the primary tumors and KTs $(r<0.3$, Figures 5(c) and 5(e)).

We also compared $\mathrm{T}$ cell densities in the primary CRCs to those in the KTs $(n=28)$. Similarly, the densities of $\mathrm{CD}^{+}, \mathrm{CD}^{+}$, and $\mathrm{FOXP}^{+}$cells were significantly higher in the primary CRCs than in the KTs $(P<0.001$; Figure $5(\mathrm{~b}))$. $\mathrm{CD} 8^{+}$cells were positively correlated between the primary tumors and KTs $(r=0.6216, P=0.0005$; Figure $5(\mathrm{~g}))$. $\mathrm{CD}^{+}$ cells and $\mathrm{FOXP}^{+}$cells were not correlated between the primary tumors and KTs (Figures 5(f) and 5(h)).

\section{Discussion}

Given the success of PD-L1/PD-1 inhibitors in metastatic tumors, we conducted this study to characterize the role of PD-L1 and the immune microenvironment in KTs. In KTs from GCs, positive tumor PD-L1 expression was associated with poor prognosis. In contrast, in KTs from CRCs, positive tumor PD-L1 expression was associated with an improved prognosis compared to negative PD-L1 expression. The GCs and CRCs had patterns of $\mathrm{CD}^{+}$cells that were correlated between the primary tumors and KTs from the same case.

Although metastatic spread is the main cause of cancerrelated death, patients with metastases have heterogeneous survival outcomes. For KTs, survival is associated with the primary tumor $[14,15]$. In our study, the CRC patients showed a longer OS time ( $17 \pm 2.397$ months) than the GC patients ( $9 \pm 1.7$ months), which was consistent with previous studies $[14,15]$. To address the prognostic value of tumor 


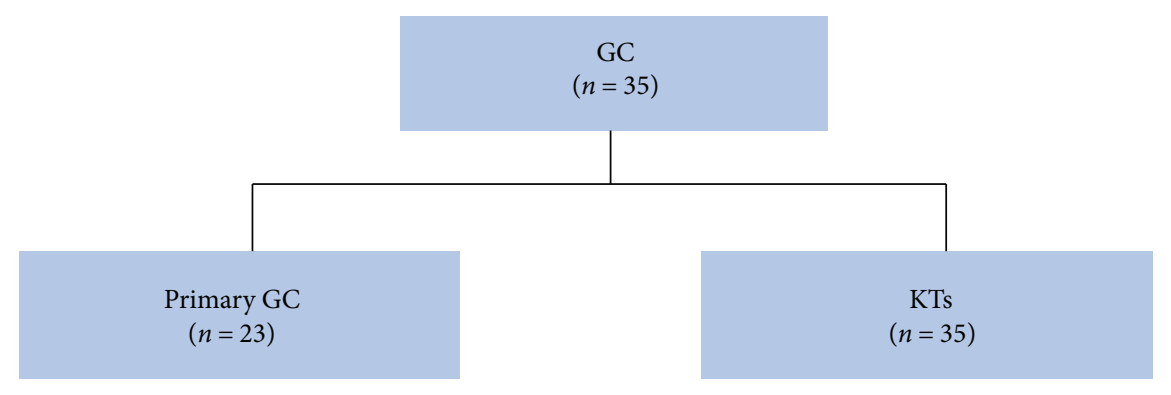

(a)

OS based on tumor PD-L1 in primary GC
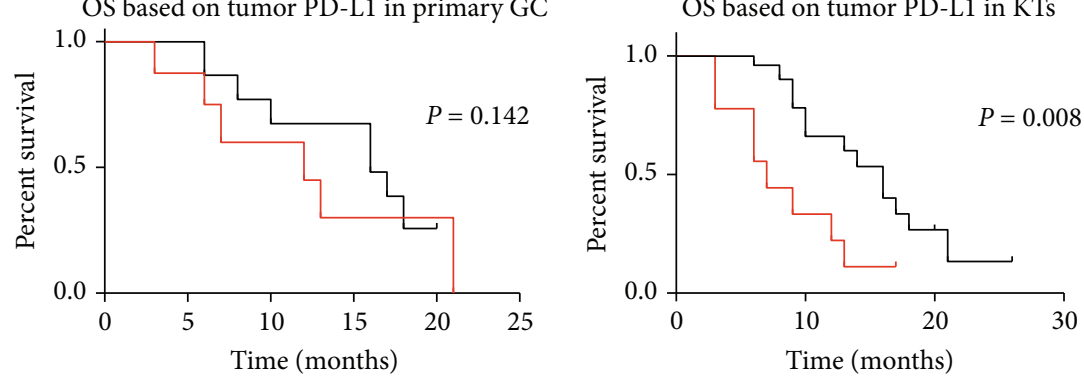

+ Negative

- Negative

- Positive

(b)

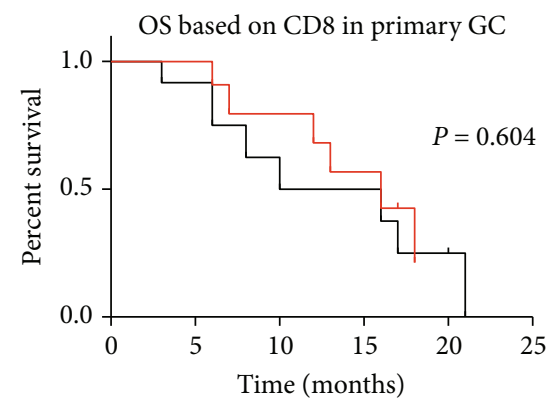

(c)

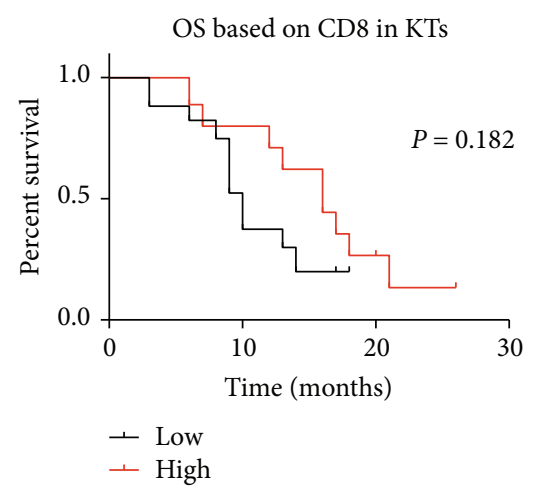

(d)

(e)

OS based on PD-L1 and CD8 in primary GC

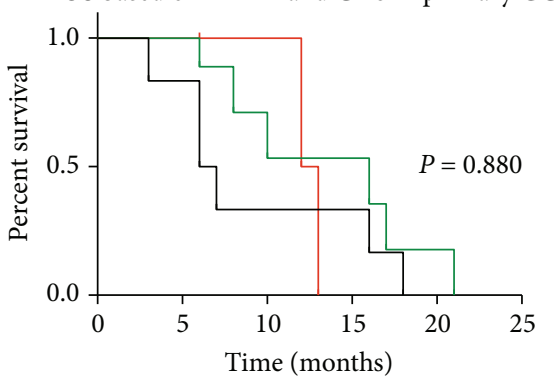

OS based on PD-L1 and CD8 in KTs

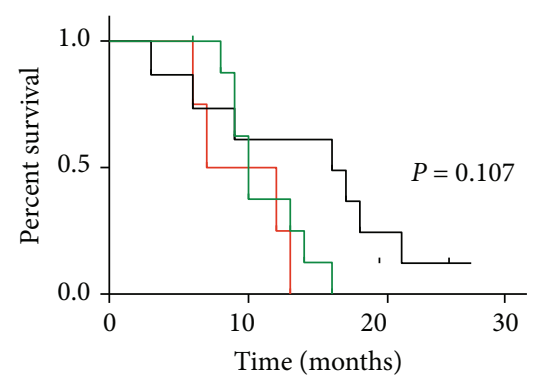

$$
\begin{aligned}
& -\mathrm{PD}-\mathrm{L} 1^{\text {neg }} / \mathrm{CD} 8^{\text {low }} \\
& \text { - } \mathrm{PD}-\mathrm{L} 1^{\text {pos }} / \mathrm{CD} 8^{\text {low }} \\
& \text { or PD-L1 } 1^{\text {neg }} / \mathrm{CD}^{\text {h }}{ }^{\mathrm{h}} \\
& - \text { PD-L1 }{ }^{\text {pos }} / \mathrm{CD}^{\text {hi }}
\end{aligned}
$$

$$
\begin{aligned}
& + \text { PD-L1 }{ }^{\text {neg }} / \mathrm{CD} 8^{\text {low }} \\
& + \text { PD-L1 } 1^{\text {pos }} / \mathrm{CD} 8^{\text {low }} \\
& \text { or PD-L1 } 1^{\text {neg }} / \mathrm{CD}^{\text {hi }} \\
& + \text { PD-L1 }^{\text {pos }} / \mathrm{CD} 8^{\text {hi }}
\end{aligned}
$$

(f)

(g)

FIgure 2: Prognostic value of tumor PD-L1 expression and CD8 T cell densities in the primary GCs and KTs. Sample cohorts (a). KaplanMeier survival curves for OS according to the PD-L1 expression in primary GCs (b) and KTs (c). Kaplan-Meier survival curves for OS according to the CD8 T cell densities in primary GCs (d) and KTs (e). Kaplan-Meier survival curves for OS according to a combined analysis of the PD-L1 expression and CD8 T cell densities in primary GCs (f) and KTs (g). 


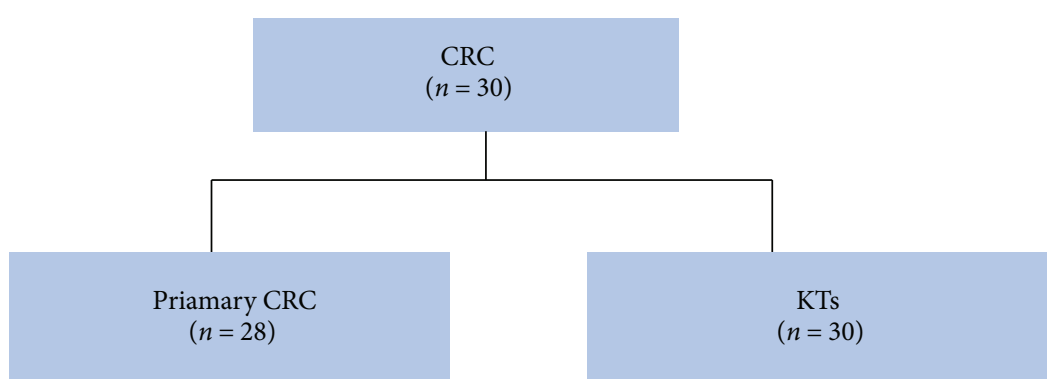

(a)

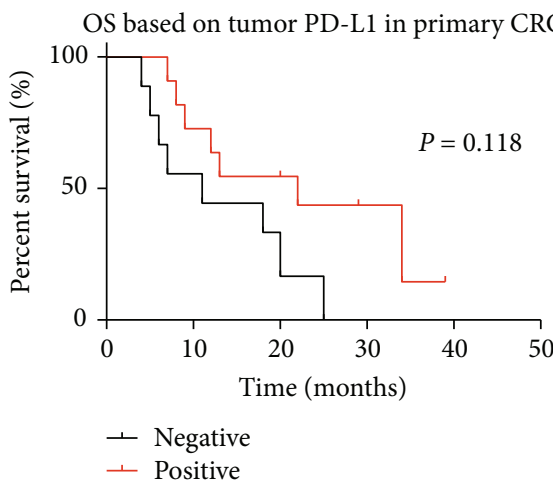

(b)

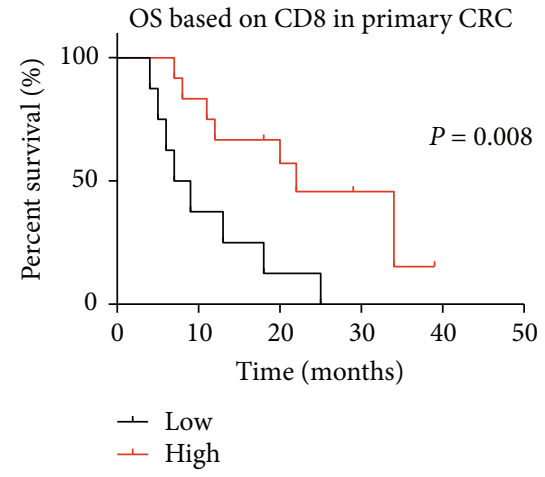

(d)

OS based on PD-L1 and CD8 in primary CRC

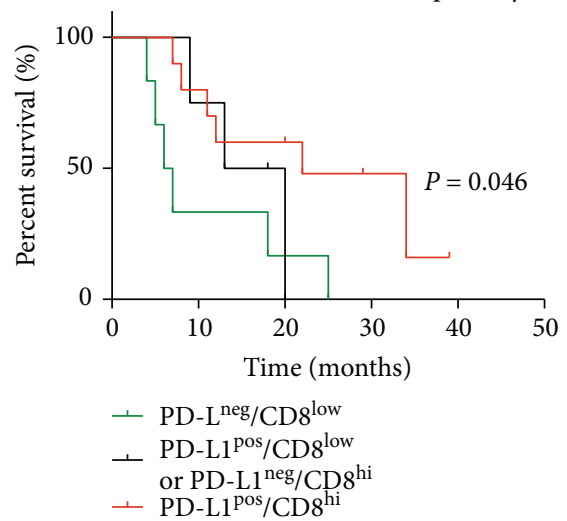

(f)

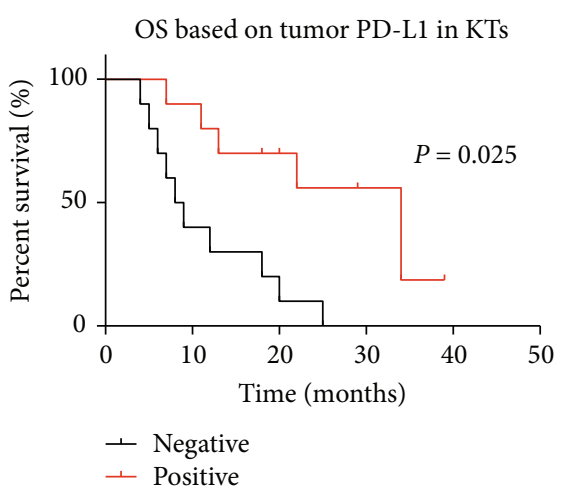

(c)

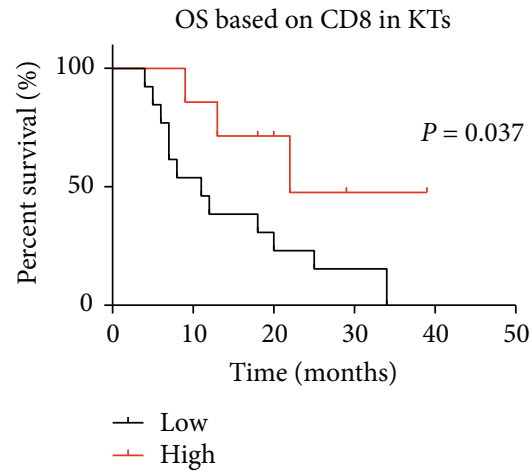

(e)

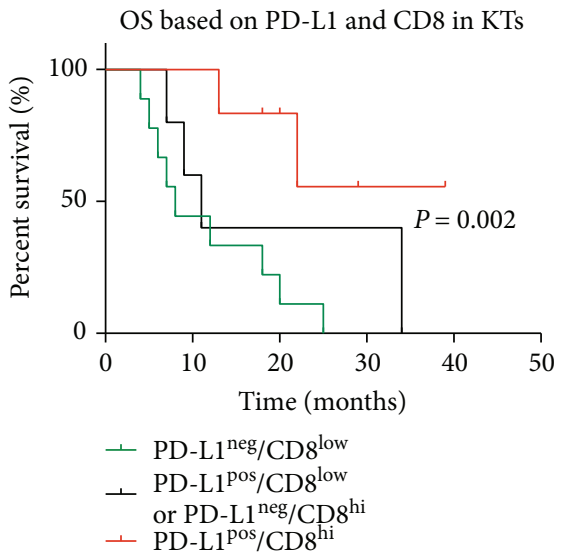

(g)

Figure 3: Prognostic value of tumor PD-L1 expression and CD8 T cell densities in the primary CRCs and KTs. Sample cohorts (a). KaplanMeier survival curves for OS according to the PD-L1 expression in primary CRCs (b) and KTs (c). Kaplan-Meier survival curves for OS according to the CD8 T cell densities in primary CRCs (d) and KTs (e). Kaplan-Meier survival curves for OS according to a combined analysis of the PD-L1 expression and CD8 T cell densities in primary CRCs (f) and KTs (g). 
TABLE 1: Univariate and multivariate analysis for prognostic factors affecting OS in GC.

\begin{tabular}{|c|c|c|c|c|c|}
\hline \multirow{2}{*}{ Risk factors } & \multicolumn{2}{|c|}{ Univariate analysis } & \multirow{2}{*}{ Risk factors } & \multicolumn{2}{|c|}{ Multivariate analysis } \\
\hline & HR $(95 \%$ CI $)$ & $P$ & & $\mathrm{HR}(95 \% \mathrm{CI})$ & $P$ \\
\hline Menopause status: menopause & $2.860(1.057-7.736)$ & 0.038 & Menopause status: menopause & $2.609(0.848-8.033)$ & 0.095 \\
\hline Tumor PD-L1 expression: positive & $3.201(1.273-8.050)$ & 0.013 & Tumor PD-L1 expression: positive & $2.413(0.905-6.437)$ & 0.078 \\
\hline $\mathrm{CD}^{+}$cell density: high & $1.132(0.475-2.701)$ & 0.779 & $\mathrm{CD}^{+}$cell density: low & $2.003(0.776-5.171)$ & 0.151 \\
\hline $\mathrm{CD}^{+}$cell density: low & $1.774(0.709-4.438)$ & 0.220 & & & \\
\hline $\mathrm{FOXP}^{+}$cell density: high & $0.964(0.405-2.294)$ & 0.934 & & & \\
\hline $\mathrm{PD}^{+}$cell density: high & $0.856(0.363-2.021)$ & 0.723 & & & \\
\hline Primary tumor size: $\geq 5 \mathrm{~cm}$ & $1.956(0.791-4.840)$ & 0.147 & & & \\
\hline Lymph node invasion: positive & $1.735(0.385-7.811)$ & 0.473 & & & \\
\hline Vascular invasion: positive & $1.393(0.576-3.365)$ & 0.462 & & & \\
\hline Neural invasion: invasion & $1.271(0.535-3.016)$ & 0.587 & & & \\
\hline Ovarian involvement: bilateral & $1.926(0.699-5.305)$ & 0.205 & & & \\
\hline
\end{tabular}

Tumor PD-L1 expression, $\mathrm{CD}^{+}$cell density, $\mathrm{CD}^{+}$cell density, $\mathrm{FOXP}^{+}$cell density, and $\mathrm{PD}^{+}$cell density are evaluated in $\mathrm{KTs}$, and variables showing $P$ values less than 0.05 are presented in italic.

TABLE 2: Univariate and multivariate analyses for prognostic factors affecting OS in CRC.

\begin{tabular}{|c|c|c|c|c|c|}
\hline \multirow{2}{*}{ Risk factors } & \multicolumn{2}{|c|}{ Univariate analysis } & \multirow{2}{*}{ Risk factors } & \multicolumn{2}{|c|}{ Multivariate analysis } \\
\hline & $\operatorname{HR}(95 \% \mathrm{CI})$ & $P$ & & $\operatorname{HR}(95 \% \mathrm{CI})$ & $P$ \\
\hline Menopause status: menopause & $2.437(0.879-6.756)$ & 0.087 & Menopause status: menopause & $3.381(1.062-10.759)$ & 0.039 \\
\hline Ratio of signet cells: high & $4.655(1.366-15.863)$ & 0.014 & Ratio of signet cells: high & $2.301(0.613-8.641)$ & 0.217 \\
\hline Tumor PD-L1 expression: negative & $5.129(1.567-16.791)$ & 0.007 & Tumor PD-L1 expression: negative & $4.451(1.062-18.658)$ & 0.041 \\
\hline Ovarian involvement: bilateral & $1.155(0.429-3.109)$ & 0.776 & $\mathrm{CD}^{+}$cell density: low & $1.816(0.462-7.140)$ & 0.393 \\
\hline $\mathrm{CD}^{+}$cell density: low & $1.640(0.606-4.436)$ & 0.330 & & & \\
\hline $\mathrm{CD}^{+}$cell density: low & $3.408(0.964-12.044)$ & 0.057 & & & \\
\hline FOXP3 $^{+}$cell density: high & $1.316(0.490-3.531)$ & 0.586 & & & \\
\hline PD $-1^{+}$cell density: high & $0.819(0.301-2.230)$ & 0.696 & & & \\
\hline Primary tumor size: $\geq 5 \mathrm{~cm}$ & $0.399(0.134-1.181)$ & 0.097 & & & \\
\hline Lymph node invasion: positive & $1.167(0.329-4.138)$ & 0.811 & & & \\
\hline Vascular invasion: positive & $0.406(0.127-1.295)$ & 0.128 & & & \\
\hline Neural invasion: positive & $3.926(0.507-30.435)$ & 0.191 & & & \\
\hline
\end{tabular}

Tumor PD-L1 expression, $\mathrm{CD}^{+}$cell density, $\mathrm{CD}^{+}$cell density, $\mathrm{FOXP}^{+}$cell density, and $\mathrm{PD}^{+}$cell density are evaluated in $\mathrm{KTs}$, and variables showing $P$ values less than 0.05 are presented in italic.

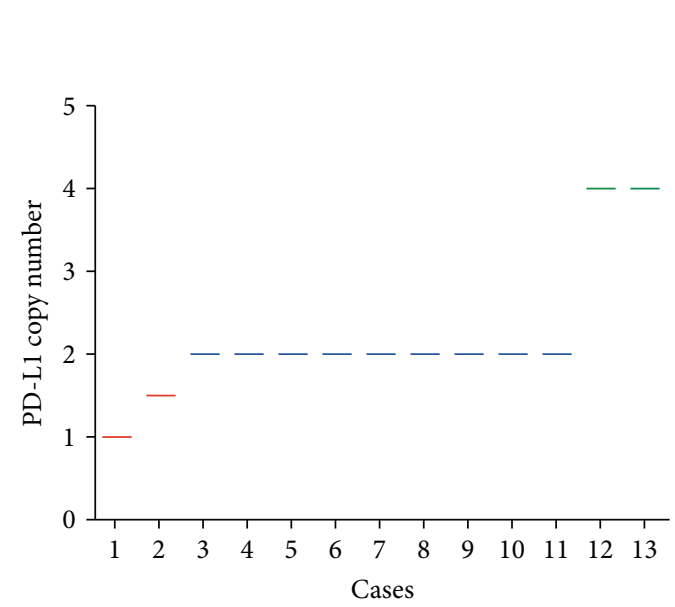

(a)

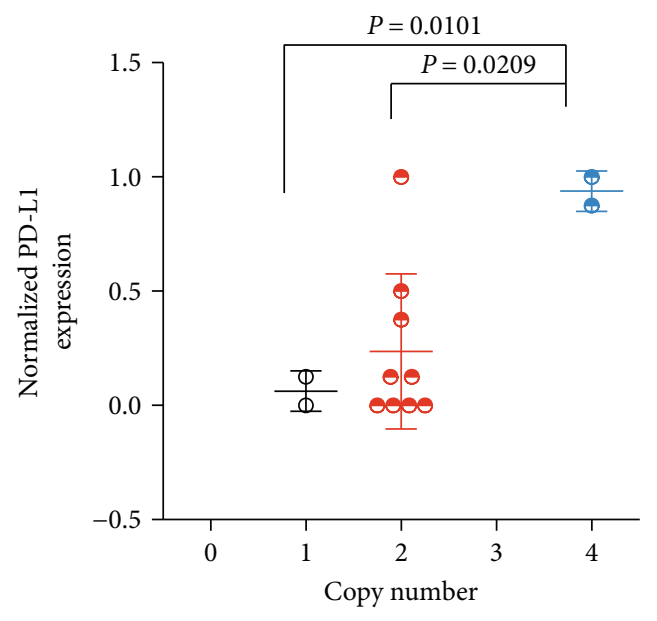

(b)

Figure 4: PD-L1 copy number status and PD-L1 expression in KTs. (a) PD-L1 copy number status in 13 KTs. (b) A higher PD-L1 expression was observed in cases with copy number gains than in normal cases $(P=0.0209)$ or cases with copy number losses $(P=0.0101)$. 


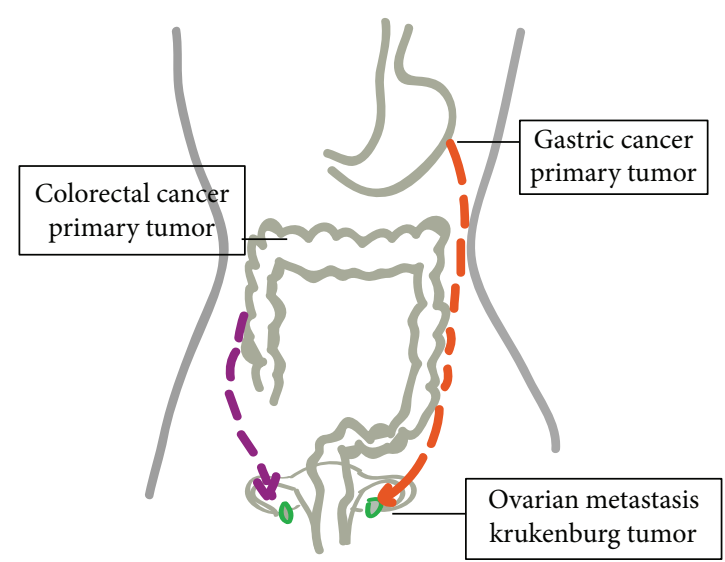

(a)

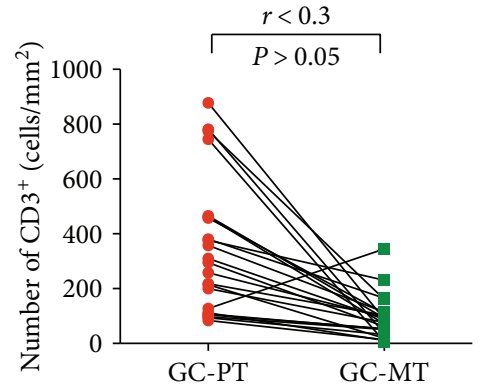

(c)

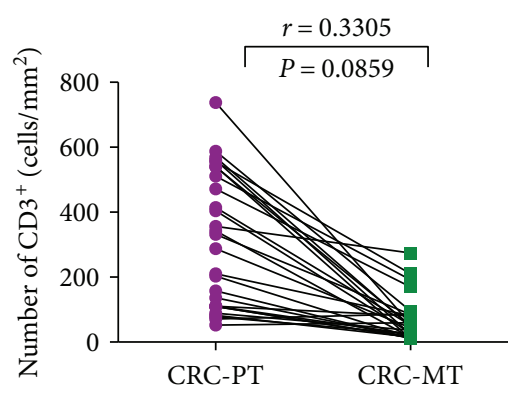

(f)

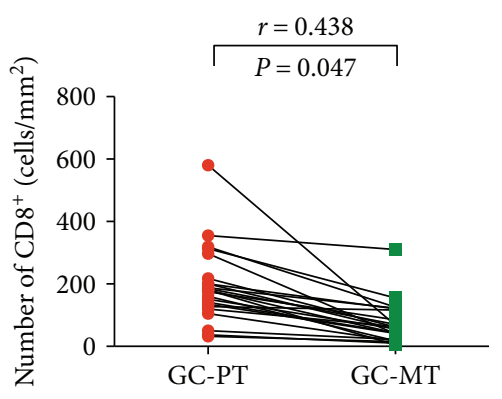

(d)

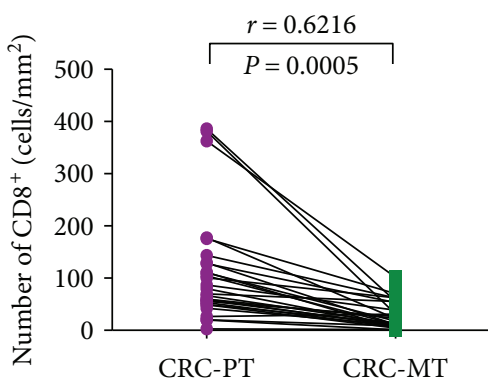

(g)

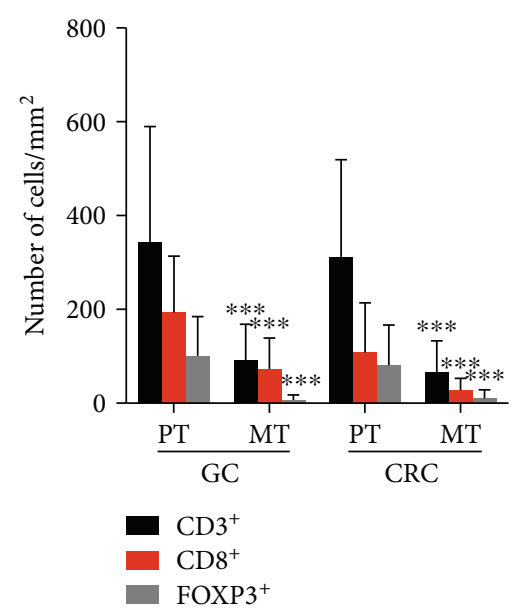

(b)

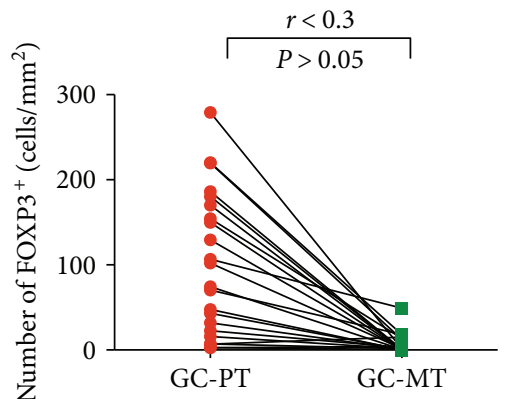

(e)

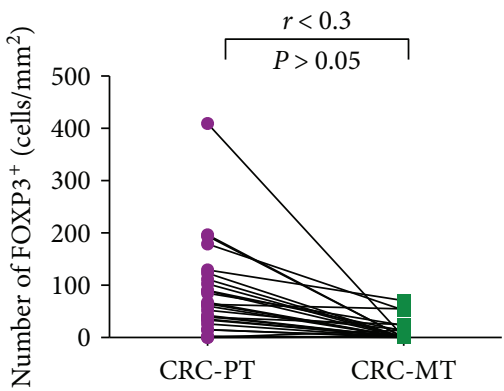

(h)

FIgURE 5: $\mathrm{CD}^{+}, \mathrm{CD}^{+}$, and $\mathrm{FOXP3}^{+}$cell densities in the primary tumor and KTs. (a) Schematic diagram of the metastasis process. (b) Primary GCs and CRCs are more infiltrated by $\mathrm{CD}^{+}, \mathrm{CD}^{+}$, and FOXP3 ${ }^{+} \mathrm{T}$ cells than the KTs. (c-e) The $R$-value shows the correlations between the primary GCs and KTs according to $\mathrm{CD}^{+}, \mathrm{CD}^{+}$, and $\mathrm{FOXP}^{+} \mathrm{T}$ cell densities. (f-h) The $R$-value shows the correlations between the primary CRCs and $\mathrm{KT}$ s according to $\mathrm{CD}^{+}, \mathrm{CD} 8^{+}$, and $\mathrm{FOXP} 3^{+} \mathrm{T}$ cell densities. ${ }^{* * *} \mathrm{P}<0.001$; PT: primary tumor; MT: metastasis.

PD-L1, we performed a Kaplan-Meier survival analysis and found that the tumor PD-L1 expression in KTs with a distinct tumor origin was associated with a different prognosis. Previous studies have demonstrated an association between high PD-L1 expression and poor prognosis in primary GC $[12,16]$, which was consistent with our study. High PD-L1 expression is reportedly associated with a better prognosis than low PD-L1 expression in several tumor types [17-19], including primary CRC; however, conflicting results have been reported for CRC $[17,20]$.

Programmed death-ligand 1 (PD-L1) expression is upregulated in multiple human cancers and attenuates the antitumor immune response [21-24]. Two mechanisms for the upregulation of PD-L1, including adaptive resistance and intrinsic immune resistance, have been proposed. Adaptive resistance occurs when $\mathrm{PD}-\mathrm{L} 1 / \mathrm{PD}-1$ serves as a negative feedback mechanism that follows $\mathrm{CD}^{+} \mathrm{T}$ cell infiltration and is driven by the immune system [13, 23, 25]. Intrinsic resistance leads to $\mathrm{PD}-\mathrm{L} 1$ upregulation due to a copy number gain, mutations, or oncogenic signaling within the tumor [26].

Genome abnormalities might affect the tumor PD-L1 expression [10]. A previous study had reported that the PD-L1 expression was significantly higher in cases with 
PD-L1 copy number gain than in normal cases in thymic carcinoma [27]. Consistent with this observation, our study showed that tumor cell PD-L1 expression was higher in cases with copy number gains. In addition to copy number aberrations, oncogenic mutations might also participate in the mechanism of PD-L1 overexpression [10]. Thus, further studies on genetic aberrations are essential to improve treatment methods.

Although DNA copy number gains play important roles in gene overexpression, $P D-L 1$ gene amplification was identified in only 2 of 13 samples, and this evidence was insufficient to explain the overexpression of PD-L1. Previous studies have reported a significant relationship between tumor PD-L1 expression and the $\mathrm{CD}^{+}$cell density $[12,28]$. IDO, PD-L1, and FOXP $3^{+}$regulatory $\mathrm{T}$ cell inhibitory pathways might serve as a negative feedback mechanism that follows $\mathrm{CD}^{+} \mathrm{T}$ cell infiltration [13]. Another study has shown a significant inverse association between the tumor PD-L1 expression and FOXP3 ${ }^{+}$cell density [29]. However, we observed no significant association between the tumor PD-L1 expression or stromal PD-L1 expression and $\mathrm{CD}^{+}$, $\mathrm{CD}^{+}$, or $\mathrm{FOXP}^{+} \mathrm{T}$ cell density, which may be due to differences in the study populations.

We further evaluated the immune contexture of the primary tumors and KTs. From the primary tumors to the metastases, the GCs and CRCs had significant correlation patterns between $\mathrm{CD}^{+}$cells. Consistently, previous studies have demonstrated that the immune contexture in the primary tumor results in "educated" immune cells that are recalled at the metastatic sites [30]. Tumor cells reportedly imprint their microenvironments during all disease stages, with a similar architecture and clinical impact [30-32]. Our study implies that the immune contexture may be partially reproducible from the primary tumor to metastatic tumors and may affect tumor PD-L1 expression.

Our study has some limitations. First, most of the KTs were over $5 \mathrm{~cm}$ in size and most were associated with no normal tissues in the margins. Thus, we were not able to assess the tumor and stromal PD-L1 expressions and the immune cell infiltration within the tumor-invasive margin. Second, the study included a small sample size because (i) Krukenberg tumor is uncommon, only accounting for $1 \%$ to $2 \%$ of all ovarian tumors [5]; (ii) whether an ovarian surgical resection should be performed has not been established. Samples are difficult to obtain. However, more samples are needed to confirm our hypothesis.

In conclusion, positive tumor PD-L1 expression implies that there might be implications for targeting the PD-L1/ $\mathrm{PD}-1$ axis in KTs. The prognostic value and immune pattern in the metastases might be useful for guiding treatment.

\section{Conclusions}

Our results characterized the role of PD-L1 and the immune microenvironment in KTs. Tumor PD-L1 expression predicted diverse prognoses in KTs with different corresponding origins. In KTs from GCs, positive tumor PD-L1 expression was associated with poor prognosis. In contrast, in KTs from CRCs, the positive tumor PD-L1 expression was associated with an improved prognosis compared to the negative PD-L1 expression. The GCs and CRCs had patterns of $\mathrm{CD}^{+}$cells that were correlated between the primary tumors and KTs from the same case, indicating that the primary tumor exerted an influence on the immune environment in the metastases. It further highlighted targets for immunebased therapy in KTs from GCs and CRCs.

\section{Data Availability}

The data used to support the findings of this study are available from the corresponding author upon request.

\section{Conflicts of Interest}

The authors declare no conflicts.

\section{Authors' Contributions}

Haiyan Tai, Qin Yang, and Zhiyong Wu share co-first authorship.

\section{Acknowledgments}

This work was supported by funding from the National Key R\&D Program of China (2016YFC1303100) and the National Natural Science Foundation of China (81472678).

\section{Supplementary Materials}

Supplementary Table S1: correlation between the PD-L1 expression on the tumor or stroma and clinicopathological characteristics in KTs from GCs. Supplementary Table S2: correlation between the PD-L1 expression on the tumor or stroma and clinicopathological characteristics in KTs from CRCs. Supplementary Table S3: relation between the PD-L1 expression and T cell densities in KTs from GCs. Supplementary Table S4: relation between the PD$\mathrm{L} 1$ expression and $\mathrm{T}$ cell densities in KTs from CRCs. (Supplementary Materials)

\section{References}

[1] R. H. Young, "From Krukenberg to today: the ever present problems posed by metastatic tumors in the ovary: part I. Historical perspective, general principles, mucinous tumors including the Krukenberg tumor," Advances in Anatomic Pathology, vol. 13, no. 5, pp. 205-227, 2006.

[2] K. H. Kim, K. W. Lee, S. K. Baek et al., "Survival benefit of gastrectomy \pm metastasectomy in patients with metastatic gastric cancer receiving chemotherapy," Gastric Cancer, vol. 14, no. 2, pp. 130-138, 2011.

[3] J. H. Cheong, W. J. Hyung, J. Chen, J. Kim, S. H. Choi, and S. H. Noh, "Survival benefit of metastasectomy for Krukenberg tumors from gastric cancer," Gynecologic Oncology, vol. 94, no. 2, pp. 477-482, 2004.

[4] F. Erroi, M. Scarpa, I. Angriman et al., "Ovarian metastasis from colorectal cancer: prognostic value of radical oophorectomy," Journal of Surgical Oncology, vol. 96, no. 2, pp. 113117, 2007. 
[5] O. M. Al-Agha and A. D. Nicastri, "An in-depth look at Krukenberg tumor: an overview," Archives of Pathology \& Laboratory Medicine, vol. 130, no. 11, pp. 1725-1730, 2006.

[6] S. L. Topalian, C. G. Drake, and D. M. Pardoll, "Immune checkpoint blockade: a common denominator approach to cancer therapy," Cancer Cell, vol. 27, no. 4, pp. 450-461, 2015.

[7] J. R. Brahmer, C. G. Drake, I. Wollner et al., "Phase I study of single-agent anti-programmed death-1 (MDX-1106) in refractory solid tumors: safety, clinical activity, pharmacodynamics, and immunologic correlates," Journal of Clinical Oncology, vol. 28, no. 19, pp. 3167-3175, 2010.

[8] F. S. Hodi, S. J. O'Day, D. F. McDermott et al., "Improved survival with ipilimumab in patients with metastatic melanoma," The New England Journal of Medicine, vol. 363, no. 8, pp. 711-723, 2010.

[9] C. Robert, L. Thomas, I. Bondarenko et al., "Ipilimumab plus dacarbazine for previously untreated metastatic melanoma," The New England Journal of Medicine, vol. 364, no. 26, pp. 2517-2526, 2011.

[10] D. T. Le, J. N. Uram, H. Wang et al., "PD-1 blockade in tumors with mismatch-repair deficiency," The New England Journal of Medicine, vol. 372, no. 26, pp. 2509-2520, 2015.

[11] S. L. Topalian, F. S. Hodi, J. R. Brahmer et al., "Safety, activity, and immune correlates of anti-PD-1 antibody in cancer," The New England Journal of Medicine, vol. 366, no. 26, pp. 24432454, 2012.

[12] E. D. Thompson, M. Zahurak, A. Murphy et al., "Patterns of PD-L1 expression and CD8 T cell infiltration in gastric adenocarcinomas and associated immune stroma," Gut, vol. 66, no. 5, pp. 794-801, 2017.

[13] S. Spranger, R. M. Spaapen, Y. Zha et al., "Up-regulation of PD-L1, IDO, and T(regs) in the melanoma tumor microenvironment is driven by $\mathrm{CD} 8^{+} \mathrm{T}$ cells," Science Translational Medicine, vol. 5, no. 200, article 200ra116, 2013.

[14] R. Jiang, J. Tang, X. Cheng, and R. Y. Zang, "Surgical treatment for patients with different origins of Krukenberg tumors: outcomes and prognostic factors," European Journal of Surgical Oncology, vol. 35, no. 1, pp. 92-97, 2009.

[15] F. Wu, X. Zhao, B. Mi et al., "Clinical characteristics and prognostic analysis of Krukenberg tumor," Molecular and Clinical Oncology, vol. 3, no. 6, pp. 1323-1328, 2015.

[16] X. Zhang, B. Huang, L. Chen et al., “The expression status and prognostic significance of programmed cell death 1 ligand 1 in gastrointestinal tract cancer: a systematic review and metaanalysis," OncoTargets and Therapy, vol. 8, pp. 26172625, 2015.

[17] R. A. Droeser, C. Hirt, C. T. Viehl et al., "Clinical impact of programmed cell death ligand 1 expression in colorectal cancer," European Journal of Cancer, vol. 49, no. 9, pp. 22332242, 2013.

[18] K. A. Schalper, V. Velcheti, D. Carvajal et al., "In situ tumor PD-L1 mRNA expression is associated with increased TILs and better outcome in breast carcinomas," Clinical Cancer Research, vol. 20, no. 10, pp. 2773-2782, 2014.

[19] J. M. Taube, R. A. Anders, G. D. Young et al., "Colocalization of inflammatory response with B7-h1 expression in human melanocytic lesions supports an adaptive resistance mechanism of immune escape," Science Translational Medicine, vol. 4, no. 127, article 127ra37, 2012.

[20] M. Song, D. Chen, B. Lu et al., "PTEN loss increases PD-L1 protein expression and affects the correlation between PD-L1 expression and clinical parameters in colorectal cancer," PLoS One, vol. 8, no. 6, article e65821, 2013.

[21] H. Dong, S. E. Strome, D. R. Salomao et al., "Tumor-associated B7-H1 promotes T-cell apoptosis: a potential mechanism of immune evasion," Nature Medicine, vol. 8, no. 8, pp. 793800, 2002.

[22] J. A. Brown, D. M. Dorfman, F. R. Ma et al., "Blockade of programmed death-1 ligands on dendritic cells enhances $\mathrm{T}$ cell activation and cytokine production," The Journal of Immunology, vol. 170, no. 3, pp. 1257-1266, 2003.

[23] S. Inaguma, J. Lasota, A. Felisiak-Golabek et al., "Histopathological and genotypic characterization of metastatic colorectal carcinoma with PD-L1 (CD274)-expression: possible roles of tumour micro environmental factors for CD274 expression," The Journal of Pathology: Clinical Research, vol. 3, no. 4, pp. 268-278, 2017.

[24] B. C. Willis, E. A. Sloan, K. A. Atkins, M. H. Stoler, and A. M. Mills, "Mismatch repair status and PD-L1 expression in clear cell carcinomas of the ovary and endometrium," Modern Pathology, vol. 30, no. 11, pp. 1622-1632, 2017.

[25] P. C. Tumeh, C. L. Harview, J. H. Yearley et al., "PD-1 blockade induces responses by inhibiting adaptive immune resistance," Nature, vol. 515, no. 7528, pp. 568-571, 2014.

[26] M. R. Green, S. Monti, S. J. Rodig et al., "Integrative analysis reveals selective 9p24.1 amplification, increased PD-1 ligand expression, and further induction via JAK2 in nodular sclerosing Hodgkin lymphoma and primary mediastinal large B-cell lymphoma," Blood, vol. 116, no. 17, pp. 3268-3277, 2010.

[27] S. Yokoyama, H. Miyoshi, K. Nakashima et al., "Prognostic value of programmed death ligand 1 and programmed death 1 expression in thymic carcinoma," Clinical Cancer Research, vol. 22, no. 18, pp. 4727-4734, 2016.

[28] T. S. Nowicki, R. Akiyama, R. R. Huang et al., "Infiltration of CD8 T cells and expression of PD-1 and PD-L1 in synovial sarcoma," Cancer Immunology Research, vol. 5, no. 2, pp. 118-126, 2017.

[29] Y. Masugi, R. Nishihara, J. Yang et al., "Tumour CD274 (PD-L1) expression and T cells in colorectal cancer," Gut, vol. 66, no. 8, pp. 1463-1473, 2017.

[30] R. Remark, M. Alifano, I. Cremer et al., "Characteristics and clinical impacts of the immune environments in colorectal and renal cell carcinoma lung metastases: influence of tumor origin," Clinical Cancer Research, vol. 19, no. 15, pp. 40794091, 2013.

[31] N. Halama, S. Michel, M. Kloor et al., "Localization and density of immune cells in the invasive margin of human colorectal cancer liver metastases are prognostic for response to chemotherapy," Cancer Research, vol. 71, no. 17, pp. 5670-5677, 2011.

[32] S. C. Katz, V. Pillarisetty, Z. M. Bamboat et al., "T cell infiltrate predicts long-term survival following resection of colorectal cancer liver metastases," Annals of Surgical Oncology, vol. 16, no. 9, pp. 2524-2530, 2009. 


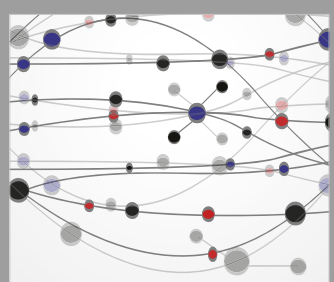

The Scientific World Journal
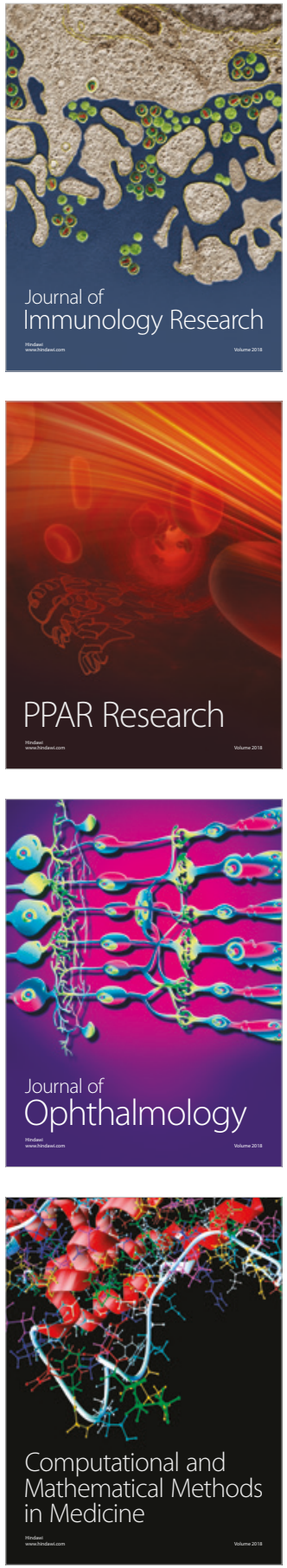

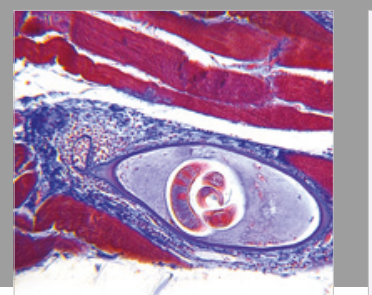

Gastroenterology Research and Practice

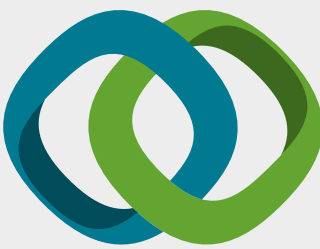

\section{Hindawi}

Submit your manuscripts at

www.hindawi.com
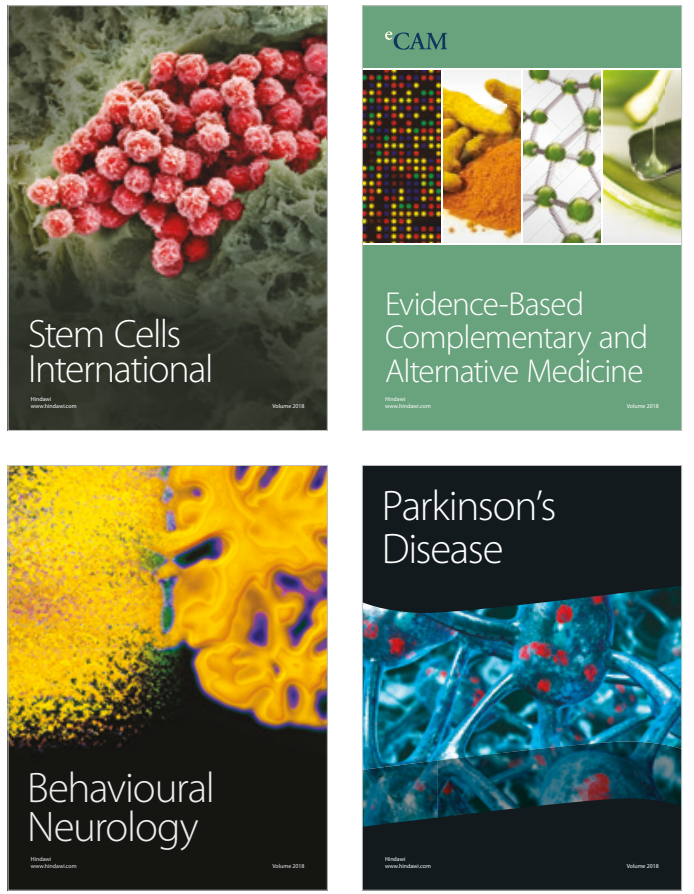

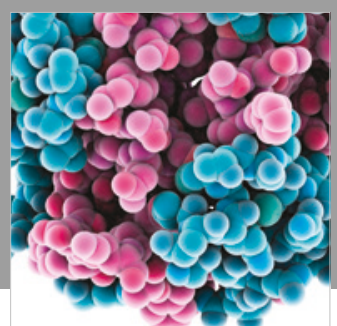

ournal of

Diabetes Research

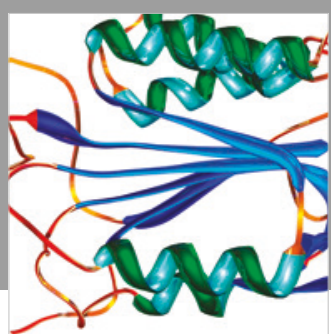

Disease Markers
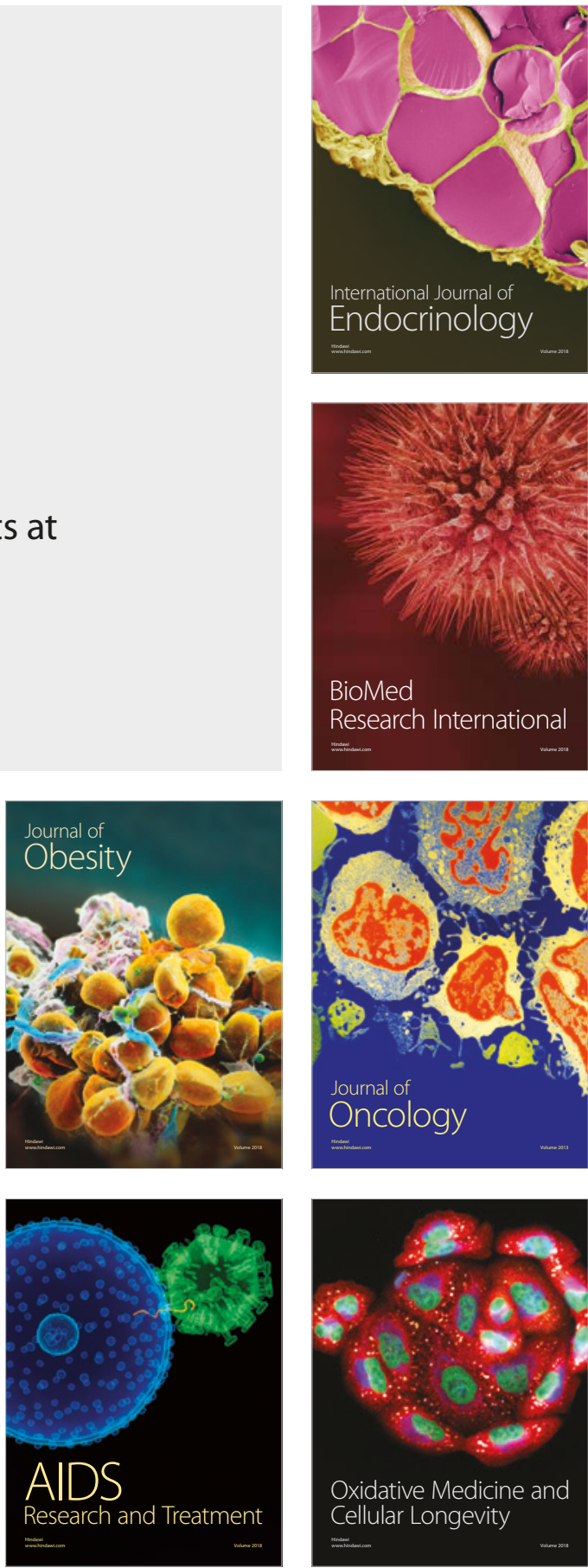